FDP-84 / ISS 2003

Submitted 29 October 2003

\title{
High-Speed Test of SFQ-Shift Register Files with PTL Wiring
}

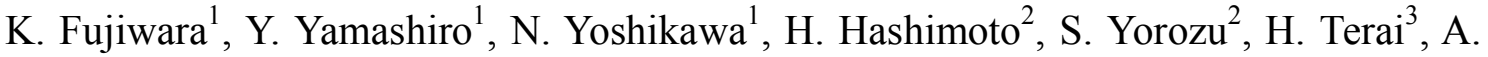
Fujimaki ${ }^{4}$

${ }^{1}$ Yokohama National University, Department of Electrical and Computer Engineering, Yokohama National University, Tokiwadai 79-5, Hodogaya, Yokohama 240-8501

${ }^{2}$ ISTEC-SRL, Tsukuba, 305-8501

${ }^{3}$ CRL, Kansai Advanced Research Center, Kobe, 651-2492

${ }^{4}$ Department of Quantum Engineering, Nagoya University, Nagoya, 464-8603

\section{Abstract}

We have been developing an SFQ shift register memory, which is one candidate to realize high-throughput and high-density superconductive memories. We have modified our memory architecture in order to adapt it to our SFQ microprocessor, CORE1. The new version of the shift register memory is composed of shift registers with nondestructive readout operation, which have an internal feedback. We have also studied the availability of passive transmission line (PTL) wiring in the memory system at high speed. The tested circuit is a 4-byte shift register file, where four kinds of wiring circuits are used between a decoder and shift registers. We have measured the dependences of 
the DC bias margin on the operating frequency for all wiring methods, and obtained almost the same dependences, which shows the availability of the PTL wiring in the memory system. We have used the NEC $2.5 \mathrm{kA} / \mathrm{cm}^{2} \mathrm{Nb}$ standard process and the CONECT cell library.

PACS code: $85.25 . \mathrm{Hv}, 85.25 . \mathrm{Na}, 85.40 . \mathrm{Bh}$

Keywords: SFQ, passive transmission line, shift register, memory

*Corresponding author.

Dr. Nobuyuki Yoshikawa

Postal address: Department of Electrical and Computer Engineering, Yokohama National University, Tokiwadai 79-5, Hodogaya, Yokohama 240-8501, Japan

Phone: +81-45-339-4259

Fax: $+81-45-338-1157$

E-mail: yoshi@yoshilab.dnj.ynu.ac.jp 


\section{Introduction}

An SFQ circuit technology is a promising digital circuit technology. It has clear advantage over the CMOS technology in terms of its high-speed and low-power operations [1]. In the realization of large-scale digital systems such as high-end SFQ network servers, the lack of a high-density and high-speed memory is one of the most serious problems [2-4].

We have been developing a shift register memory, which is one candidate to solve this problem because of its high throughput and simple circuit structure [5-7]. In this study, we have modified our memory architecture in order to adapt it to our SFQ microprocessor, CORE1. The new version of the shift register memory is composed of the shift register array with non-destructive readout operation, which has an internal feedback loop in each shift register. We will show the design details of the new memory architecture in this paper.

We have also investigated the availability of passive transmission lines (PTLs) to reduce the access time of the memory [8]. PTLs are essential components to the memory system, which can distribute the data over a large area within a short propagation delay. We have designed and implemented a 4-byte shift register file with PTL wring and compared its characteristics with one with Josephson transmission line (JTL) wiring at high speed.

\section{Memory Architecture for CORE1 Microprocessor}

In the previous report, we have shown the design details of the shift register memory system [7], which is to be used for our SFQ microprocessor, TIPPY [9]. In our previous memory design, the data-driven self-timed (DDST) architecture [10] was used to solve 
the timing problem in the clock distribution at high frequency. The system is composed of the array of the shift registers with destructive readout operation. In order to make the system with non-destructive readout operation, we added a feedback loop outside the shift register array. In this architecture, a high dense memory can be obtained due to only one feedback loop, while the throughput of the readout operation is deteriorated.

We have also been designing another type of the shift register memory for the CORE1 microprocessor [11]. CORE1 is the simplest implementation of our complexity reduced (CORE) architecture concept [12]. Both instruction and data are 8-bit-wide and bit-serial, and their bit operations are performed by a $16 \mathrm{GHz}$ local clock. The difference of its architecture from the former one is that it uses concurrent flow clocking for memory read/write operations. It also has a feedback loop in each shift register to realize non-destructive readout operation and increase the throughput of the readout operation.

Fig. 1 shows a circuit diagram of an 8-bit shift register. It uses counter flow clocking to reduce the access time. A feedback loop, composed of a non-destructive register cell (ndro), is added to realize a non-destructive readout operation. When the write pulse is inputted, the ndro is reset, and 8-bit input data are stored to the shift register. When the read pulse is inputted, the ndro is set to the on state, forming a feed back loop. In this case, the readout data are written back to the shift register through the feedback loop.

Fig. 2 shows a block diagram of the tested circuit, which is a 4-byte register file. The circuit is composed of an input buffer, a local clock generator (CG), a 2-bit packet decoder, four 8-bit shift registers and an output SFQ gate. We have designed all circuit components using the NEC Nb $2.5 \mathrm{kA} / \mathrm{cm}^{2}$ standard process and CONNECT cell library [13]. The system has four kinds of wiring circuits between a decoder and shift registers 
to investigate the influence of the PTL wiring. They are a standard JTL (a cell is $40 \mu \mathrm{m}$ square), a long JTL (a cell length is $160 \mu \mathrm{m}$ ), a short PTL (a length is $260 \mu \mathrm{m}$ ), and a long PTL (a length is $500 \mu \mathrm{m}$ ). The width and the impedance of the PTL are $34 \mu \mathrm{m}$ and $2 \Omega$, respectively. The layouts of wiring cells are shown in Fig. 3. Using a long PTL wire we can reduce the access time of the system by about 60 ps compared with the standard JTL connection.

In the write operation, first, address data are set to the decoder and a 1-bit write signal is inputted. This time the SFQ gate is set to the off state and the 8-bit shift registers are set to the write mode. Then, a 1-bit trigger pulse is inputted to the CG, and the 8-bit data stored in the input buffer are sent to the selected shift register at high-speed. In the read operation, first, address data are set and a 1-bit read signal is inputted. This time the SFQ gate is set to the on state and the shift registers are set to the read mode. Then, 8-bit trigger pulses are inputted to read the data on the shift register. This operation is nondestructive due to the internal feedback of the shift register as described above.

\section{Test results}

\subsection{8-bit shift register}

A low speed test result of the 8-bit shift register is shown in Fig.4, where rising edges in the input signals correspond to the input of SFQ pulses and transitions in the output signal are the output of SFQ pulses. Write and read pulses are not shown in the figure. From the low speed test, the DC bias margin is found to be $-25.0 \% \sim+25.1 \%$, which agrees with simulation results quite well.

\subsection{4-Byte register file with PTL wiring}


Fig. 5 shows the sequence for the high-speed test of the memory system, when the address $(1,1)$ is selected and the data $\mathrm{D}[1,1]=(10100000)$ are loaded. $\mathrm{D}[\mathrm{A} 0, \mathrm{~A} 1]$ denotes 8-bit data on the shift register specified by an address [A0, A1]. When the 1-bit trigger pulse (Trg) is inputted to the $\mathrm{CG}$, it generates 8-bit high-speed pulses and 8-bit data are sent to the selected shift register. In the test sequence, the non-destructive readout operation is confirmed by reading the same shift register twice. Fig. 6 shows test results, where successive readout operations of $\mathrm{D}[1,1], \mathrm{D}[1,1]$ are demonstrated successfully. The system contains about 1780 Josephson junctions and its circuit size is $1,200 \mu \mathrm{m} \times 1,880 \mu \mathrm{m}$. Fig. 7 shows the dependence of the DC bias margins on the operating frequency for the different wiring methods. The maximum clock frequency was found to be about $33 \mathrm{GHz}$. It agrees well with the simulation result. In the lower clock frequency, the standard JTL wiring method shows somewhat wider margins, but we can say that enough margins are obtained for all wiring methods.

\section{Conclusion}

We have modified our shift register memory architecture in order to adapt it to our CORE1 microprocessor. We have also studied the availability of the PTL wiring using a 4-byte shift register file. The results indicate that no remarkable deterioration of DC bias margin is observed in the PTL wring. In the next phase, we will scale up a memory size using the PTL wiring.

\section{Acknowledgements}

This work was supported by the New Energy and Industrial Technology Development Organization (NEDO) through ISTEC as Collaborative Research and 
Superconductors Network Device Project. 


\section{References}

[1] K.K. Likharev and V.K. Semenov, IEEE Trans. Appl. Superconduct. 1 (1991) 1.

[2] S. V. Polonsky, A. F. Kirichenko, V. K. Semenov, and K. K. Likharev, IEEE Trans. Appl. Superconduct. 5 (1995) 3000.

[3] S. Nagasawa, Y. Hashimoto, H. Numata, and S. Tahara, IEEE Trans. Appl. Superconduct. 5 (1995) 2447.

[4] Alex F. Kirichenko, Saad Sarwana, Darren K. Brock, and Masoud Radpavar, IEEE Trans. Appl. Superconduct. 11 (2001) 537.

[5] P. Yuh, and Oleg A. Mukhanov, IEEE Trans. Appl. Superconduct. 2 (1992) 214.

[6] K. Fujiwara, H. Hoshina, J. Koshiyama and N. Yoshikawa, Physica C, 378-381 (2002) 1475.

[7] Fujiwara, Y. Yamashiro, N. Yoshikawa, A. Fujimaki, H. Terai and S. Yorozu, to be published in Supercond. Sci. Technol.

[8] PTL

[9] N. Yoshikawa, F. Matsuzaki, N. Nakajima, K. Fujiwara, K. Yoda and K. Kawasaki, IEEE Trans. Appl. Superconduct., 13 (2003) 441.

[10] Z. J. Deng, N. Yoshikawa, S. R. Whiteley and T. Van Duzer, IEEE Trans. Appl. Superconduct., 7 (1997) 3830.

[11] M. Tanaka, F. Matsuzaki, T. Kondo, N. Nakajima, Y. Yamanashi, H. Terai, S. Yorozu, N. Yoshikawa, A. Fujimaki, and H. Hayakawa, Physica C, 392-396 (2003) 1490.

[12] A. Fujimaki, Y. Takai and N. Yoshikawa, IEICE Trans. Electron., E85-C (2002) 612. 
[13] S. Yorozu, Y. Kameda, H. Terai, A. Fujimaki, T. Yamada and S. Tahara, Physica C, 378-381 (2002) 1471. 


\section{Figure Captions}

Fig. 1. A block diagram of an 8-bit shift register with a feedback loop.

Fig. 2. A block diagram of a 4-byte shift register memory system for the CORE1 microprocessor. We have used four kinds of wiring circuits between a decoder and shift registers. Path "a" uses a long PTL (a length is $500 \mu \mathrm{m}$ ), path "b" uses a short PTL (a length is $260 \mu \mathrm{m}$ ), path "c" uses a long JTL (a cell length is $160 \mu \mathrm{m}$ ), and path "d" uses standard JTL (a cell is $40 \mu \mathrm{m}$ square).

Fig. 3. Mask layouts of (a) a PTL, (b) a long JTL cell and (c) a standard JTL cell.

Fig. 4. A low speed test result of the 8-bit shift register.

Fig. 5. A sequence for the high-speed test of the 4-byte shift register memory system, when address $(1,1)$ is selected.

Fig. 6. Output wave form for the successive readout of $\mathrm{D}[1,1], \mathrm{D}[1,1]$ at high-speed. The input data sequence is described in Fig. 5.

Fig. 7. The dependence the DC bias margin on the operation frequency for different wiring methods. 


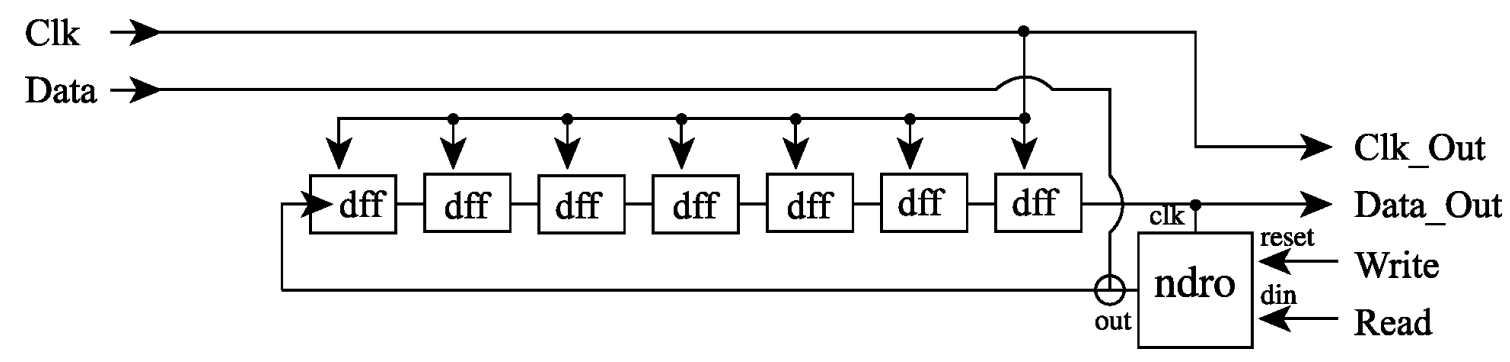

Fig. 1 


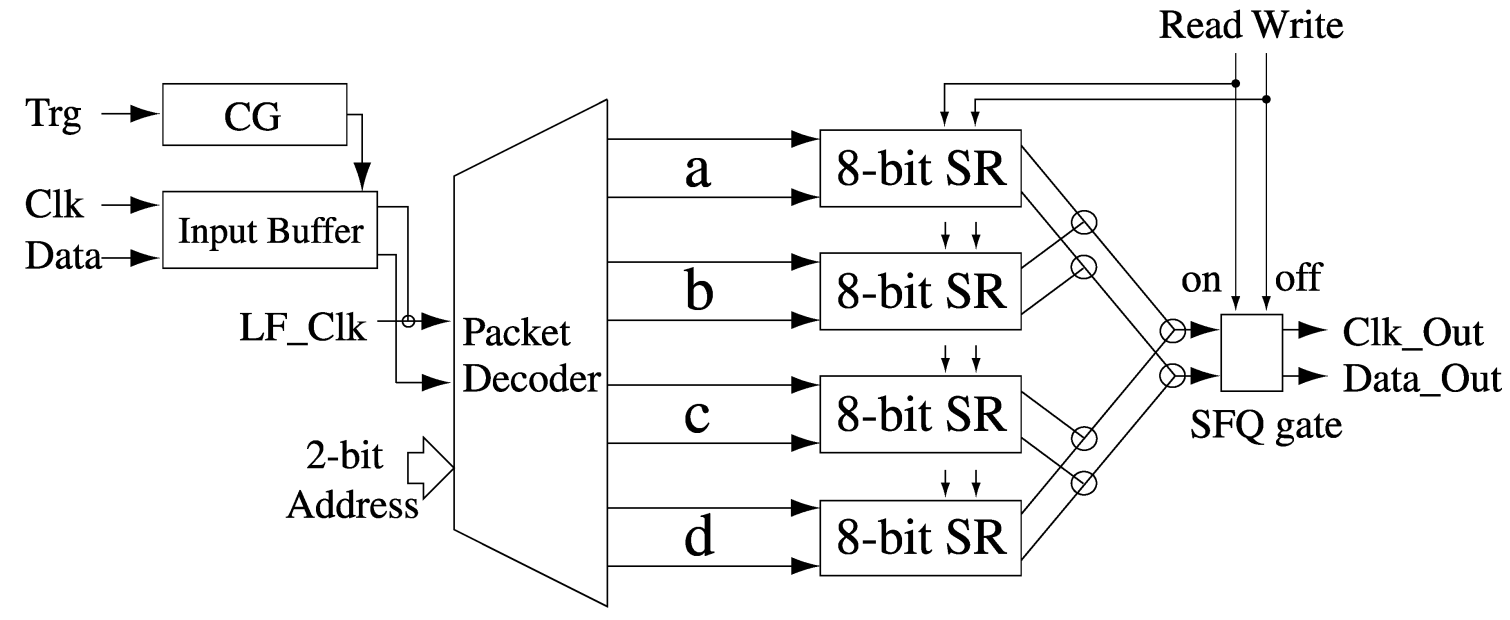

Fig. 2 


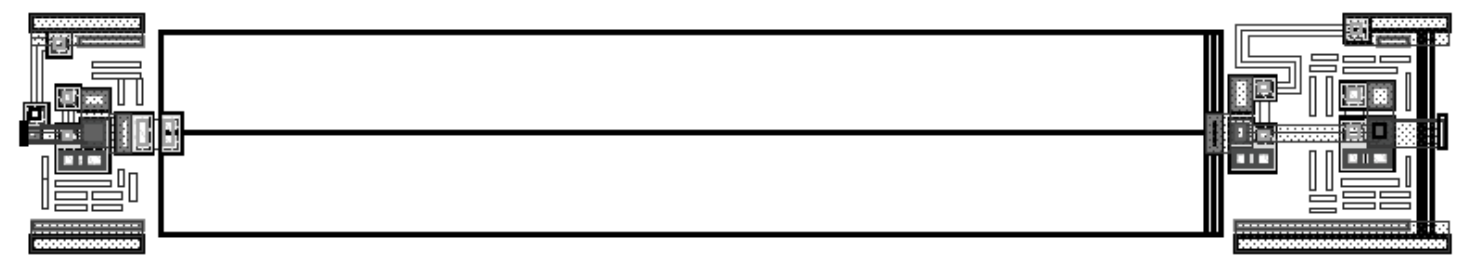

(a)

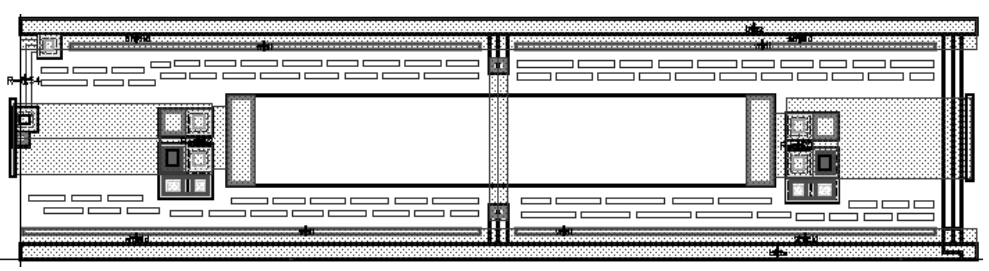

(b)

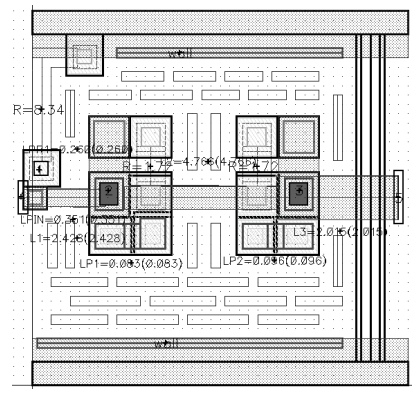

(c)

Fig. 3 


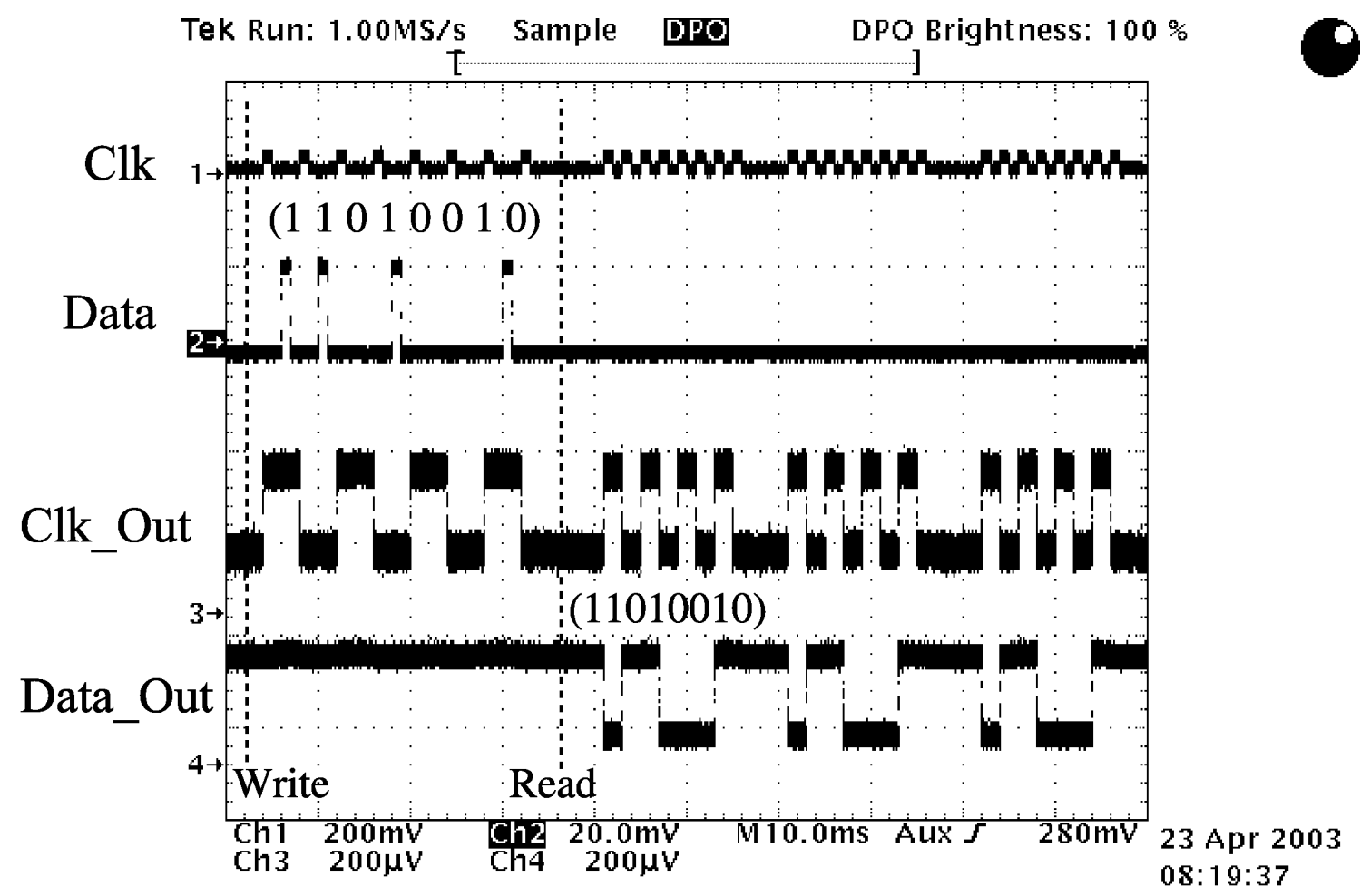

Fig. 4 


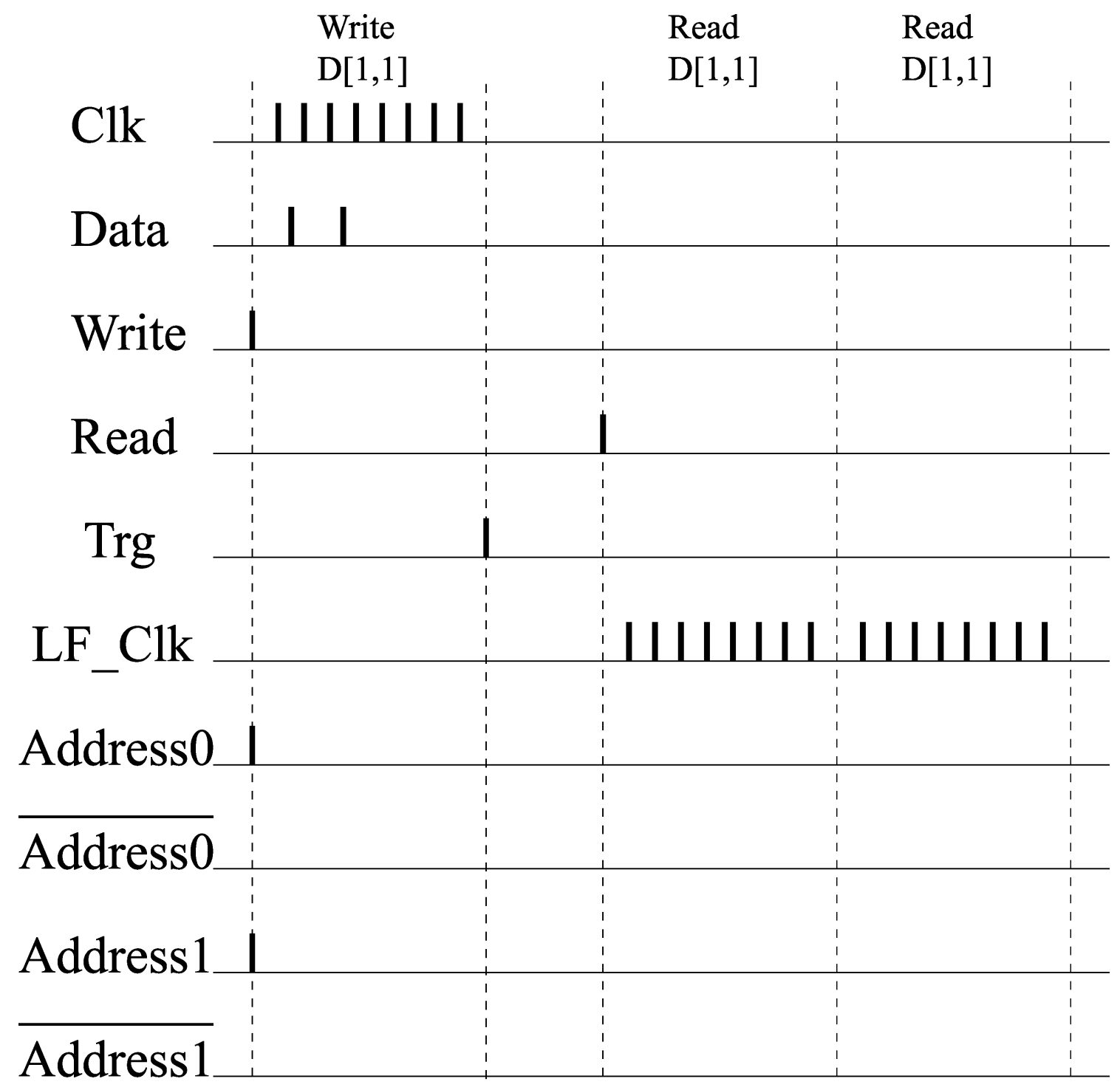

Fig. 5 


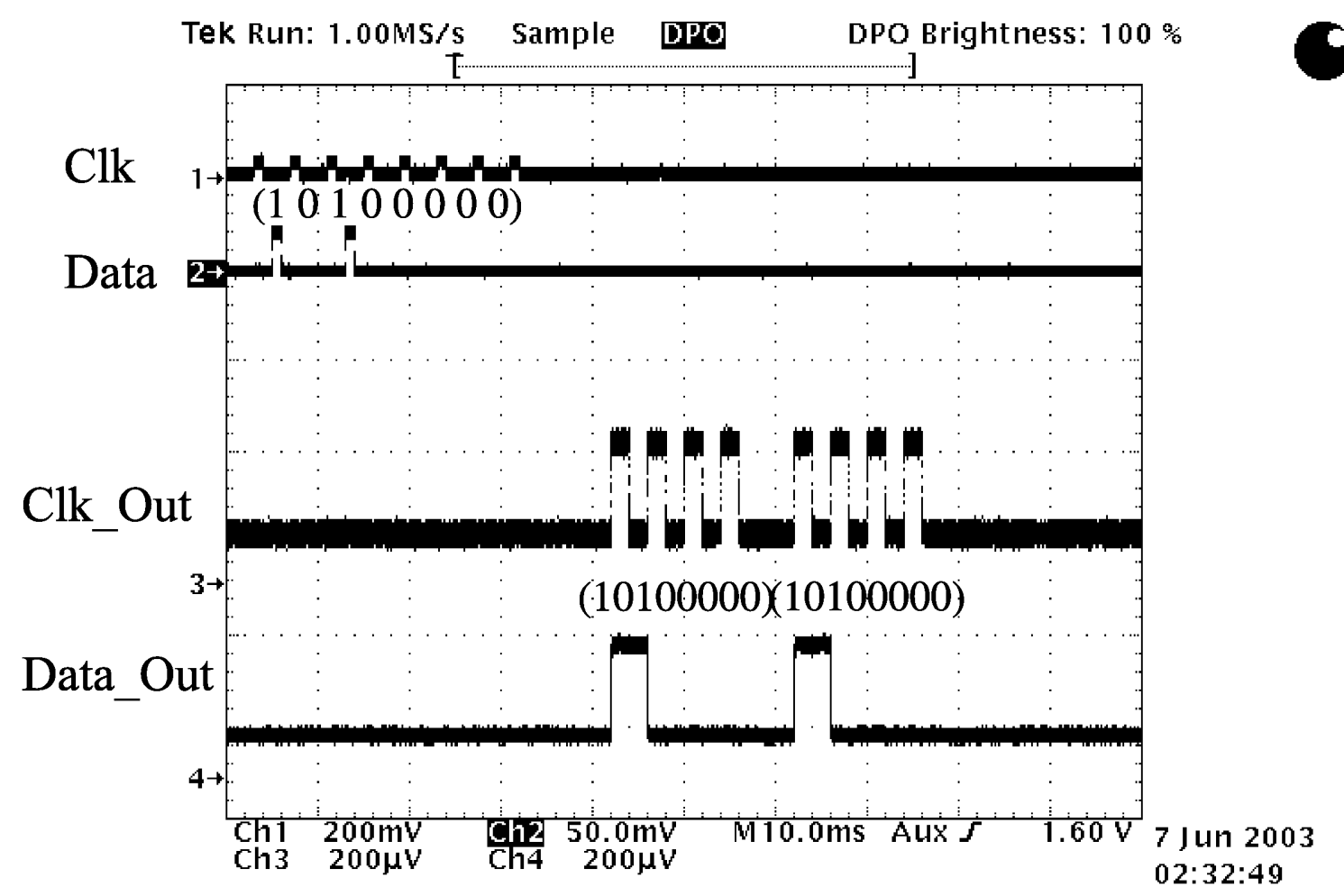

Fig. 6 


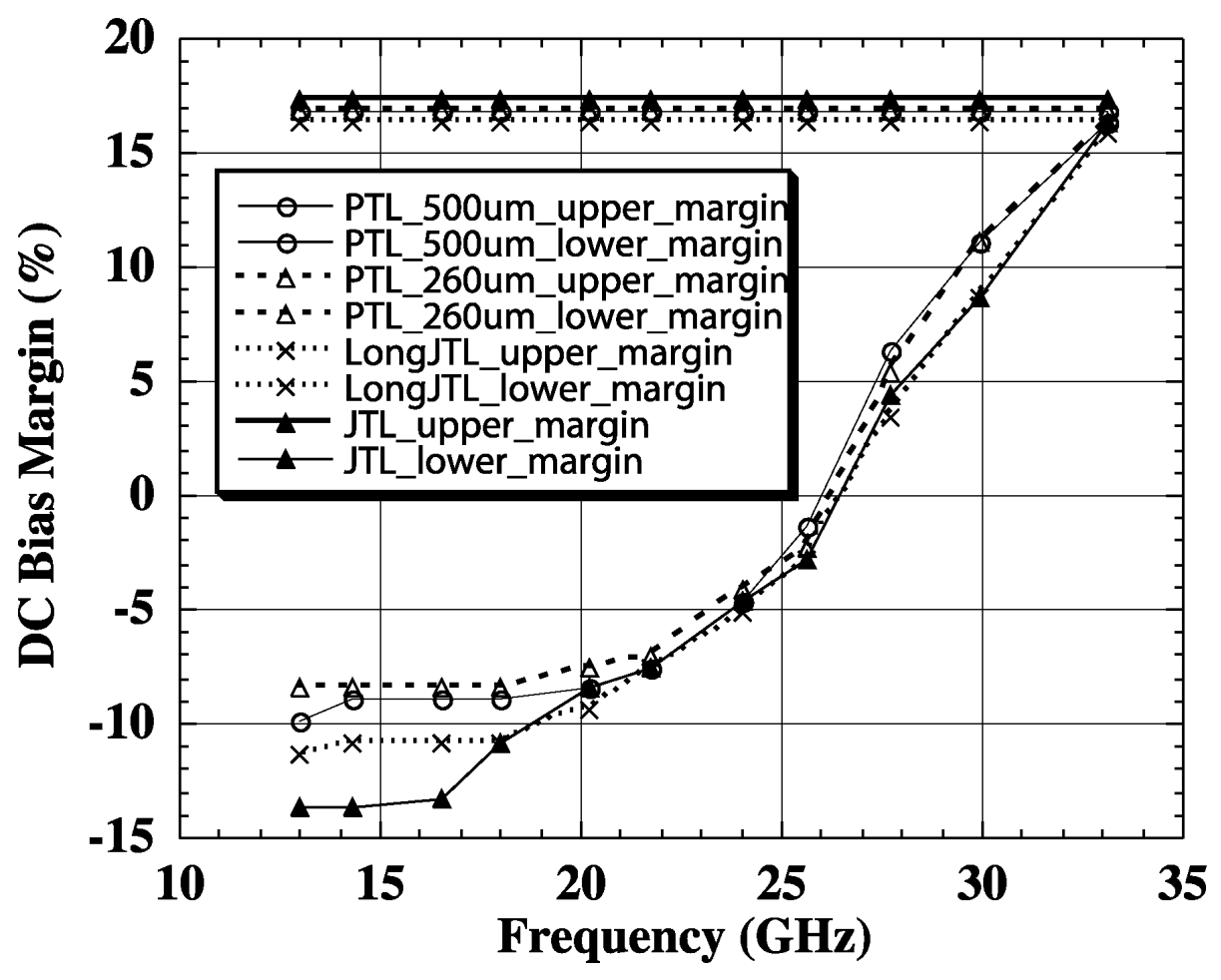

Fig. 7 